\title{
AFECTACIÓN PSICOLÓGICA DE LA POBLACIÓN RIOBAMBEÑA DURANTE EL CONFINAMIENTO POR COVID-19
}

\author{
PSYCHOLOGICAL AFFECTION OF THE RIOBAMBIAN POPULATION DURING THE \\ CONFINEMENT BY COVID-19
}

\author{
Nathaly Tixi Berrones $^{(1)}$; Luis Quijosaca Cajilema ${ }^{(2)}$; Evelyn Camacho Abarca ${ }^{(1)}$; Sara \\ Quishpe Chirau ${ }^{(1)}$; Gabriela Llerena Flores ${ }^{(1)}$. \\ (1) Universidad Nacional de Chimborazo, Riobamba-Ecuador. \\ (2) Unidad Educativa Dr. Gabriel García Moreno, Riobamba - Ecuador.
}

Email:nathalytsilvana@hotmail.com

https://doi.org/10.33789/talentos.8.2.152

\begin{abstract}
Resumen: Las medidas dictadas por el gobierno nacional durante la fase de confinamiento de la COVID-19 generaron cambios bruscos y radicales en el comportamiento humano. Las personas se vieron privadas de realizar actividades, hasta ese momento, cotidianas. Esta situación generó afectación psicológica en la población aún no estudiada ni identificada adecuadamente. El objetivo de la presente investigación fue identificar las alteraciones psicológicas que se presentaron en la población riobambeña durante la fase de confinamiento por la COVID-19. Para esto se realizó una investigación básica, descriptiva y cualitativa que tuvo como universo 684 personas adultas de la parroquia Lizarzaburu de la ciudad de Riobamba, provincia Chimborazo, Ecuador. La muestra quedó conformada por 247 personas a las que se les aplicó un cuestionario de investigación para identificar alteraciones psicológicas. Los principales resultados muestran predominio de personas con afectación psicológica $(72,47 \%)$, donde la ansiedad (37,43\%), los trastornos del sueño (29,05\%) y los dolores musculares (27,37\%) fueron las afectaciones que con mayor frecuencia fueron reportadas. Se concluye que el periodo de confinamiento por la COVID-19 generó afectación psicológica en las personas pertenecientes a la parroquia Lizarzaburu de la ciudad de Riobamba. La no existencia de esquemas terapéuticos y preventivos de la enfermedad fue señalada como la principal causa para la aparición de
\end{abstract}


trastornos psicológicos. Es necesario consolidar servicios de salud mental para hacer frente a las manifestaciones de afectación psicológicas generadas durante la fase de confinamiento obligatorio por la COVID-19.

Palabras clave: Ansiedad, Coronavirus, COVID-19, Depresión, Trastornos Psicológicos

\begin{abstract}
The measures dictated by the national government during the confinement phase of COVID-19 generated abrupt and radical changes in human behavior. People were deprived of activities, until then, daily. This situation generated psychological affectation in the population not yet studied or adequately identified. The objective of this research was to identify the psychological alterations that occurred in the riobambeñ population during the confinement phase due to COVID-19. For this, a basic, descriptive and qualitative research was carried out that had as a universe 684 adults from the Lizarzaburu parish of the city of Riobamba, Chimborazo province, Ecuador. The sample consisted of 247 people to whom a research questionnaire was applied to identify psychological disorders. The main results show a predominance of people with psychological affectation (72.47\%), where anxiety (37.43\%), sleep disorders (29.05\%) and muscle pain (27.37\%) were the affectations that were most frequently reported. It is concluded that the period of confinement due to COVID-19 generated psychological affectation in the people belonging to the Lizarzaburu parish in the city of Riobamba. The non-existence of therapeutic and preventive schemes for the disease was indicated as the main cause for the appearance of psychological disorders. It is necessary to consolidate mental health services to face the manifestations of psychological affectation generated during the phase of compulsory confinement by COVID-19.
\end{abstract}

Keywords: Anxiety, Coronavirus, COVID-19, Depression, Psychological Disorders

\title{
I. INTRODUCCIÓN
}

A finales del año 2019 se identifica por primera vez en la historia, la presencia de una nueva enfermedad en la ciudad China de Wuhan. La enfermedad, causada por el coronavirus SARS-CoV-2, fue denominada como COVID-19 haciendo alusión al agente causal y al año de identificación de la enfermedad (Solis Cartas y Martínez, 2020).

La COVID-19 tuvo una rápida propagación a nivel mundial, afectando los 5 continentes. Adicionalmente generó en la población afectada elevados índices de contagio, complicaciones y muertes; lo que sumado al desconocimiento sobre medidas preventivas y terapéuticas y la no existencia de vacunas u otros medios que frenaran la propagación de la enfermedad motivaron que la Organización Mundial de la Salud (OMS) considerara a la COVID-19 como una pandemia solo tres 
meses después de conocida su presencia (Solis y Martínez, 2020).

A partir de la comunicación realizada por la OMS los directivos gubernamentales a nivel mundial consideraron necesario implementar acciones y medidas para frenar el contagio de la COVID-19 y minimizar de esta forma la morbimortalidad por la enfermedad. Ecuador fue uno de los primeros países en reportar la presencia de COVID-19. A este reporte le siguieron una ola de contagios comenzando por la provincia de Guayas que colapso el sistema de salud nacional y generó una situación de crisis en todo el país motivada por el aumento descontrolado y desenfrenado de reportes de casos en todas las provincias del país (Solis, 2020).

Ante esta situación el gobierno decretó una serie de medidas restrictivas encaminadas a limitar, entre otras, la movilidad y el contacto entre personas. El cierre de fronteras, el confinamiento de la población y las restricciones de movilidad vehicular $\mathrm{y}$ peatonal fueron algunas de las acciones implementadas con el afán de frenar la propagación de la COVID-19 (Solis, 2020).

Con las medidas implementadas la población ecuatoriana vio cómo su rutina diaria cambiaba de forma, tipo y actividades radicalmente. El cierre de centros laborales, la introducción del teletrabajo y clases virtuales, la restricción vehicular y peatonal y la limitación para salir de sus hogares constituyeron vivencias de los meses de marzo a junio del 2020 (Peraza, 2020).

La provincia de Chimborazo, especialmente la ciudad de Riobamba, también vivió las medidas implementadas que incluyeron el cierre total de centros laborales; imposibilidad de salir de las casas, confinamiento obligatorio y toque de queda por más de 12 horas diarias. También se incluyó la restricción de salida vehicular solo dos veces a la semana, limitación para asistir a mercados para adquirir productos de primera necesidad y cierre total de todos los locales destinados a actividades sociales. A esta situación se adicionó el cierre de escuelas y la suspensión de consultas externas en centros de atención de salud del primer y segundo nivel de atención públicos y privados (Rodríguez-Morales, 2020).

El fin de las medidas era frenar el contagio de la enfermedad. Sin embargo, el cambio brusco de actividades y la necesidad de tener que adaptarse a una nueva forma de vida con limitaciones considerables de la movilidad y orientada hacia un confinamiento obligatorio motivaron cambios psicológicos en la población con presencia de manifestaciones clínicas y trastornos conductuales que aún no han sido identificados ni cuantificados adecuadamente, lo cual ha sido reportado por Lucero y colaboradores (2021).

Diversas investigaciones han demostrado la aparición de manifestaciones de ansiedad, depresión y otros trastornos de la esfera psicológica en la población, secundario al periodo de confinamiento y el resto de medidas tomadas durante la pandemia de COVID-19; estas han afectado a la población de forma general (Bermejo-Sánchez, Peña-Ayudante, \& Espinoza-Portilla, 2020). Autores como Martínez-Pérez y colaboradores (2020), describen que la situación epidemiológica generada por la COVID-19 ha traspasado 
el plano epidemiológico y ha afectado considerablemente la esfera psicosocial, condicionando afectación emocional en distintos grados y formas de expresión. Por su parte, Leon Reyna, Lora Loza y Rodriguez Vega (2021) han señalado que la afectación psicológica generada 'por el estilo de vida que fue impuesto durante el confinamiento por COVID-19 ha repercutido negativamente en la aparición de estrés laboral, sobre todo en el personal de la salud y espacialmente en personal que realiza acciones de enfermería.

Es por eso, que teniendo en cuenta la situación vivida por la población riobambeña durante el confinamiento por la COVID-19 y la posible repercusión que las medidas tomadas hayan tenido sobre la salud mental de las personas; se decide realizar esta investigación con el objetivo de identificar las alteraciones psicológicas que se presentaron en la población riobambeña durante la fase de confinamiento por la COVID-19.

\section{MATERIALES Y MÉTODOS}

Se realizó una investigación básica, no experimental con enfoque cuantativo y alcance descriptivo. El universo estuvo constituido por un total de 684 personas adultas residentes en la parroquia Lizarzaburu de la ciudad de Riobamba, provincia Chimborazo, Ecuador. Para calcular el tamaño de la muestra se utilizó la fórmula matemática de cálculo muestral para poblaciones conocidas

Después de realizar el cálculo matemático se identificó que la muestra debía estar compuesta por 247 personas. La composición final se realizó mediante la aplicación del muestreo aleatorio simple donde cada persona presentó la misma probabilidad de formar parte de la muestra del estudio. Cada persona que formó parte del estudio cumplió los criterios de inclusión y exclusión definidos para la investigación:

Criterios de inclusión

- Personas adultas (mayores de 18 años de edad) residentes en la parroquia Lizarzaburu durante el periodo marzo-julio 2020.

- Personas que estuvieron de acuerdo en participar en el estudio y lo expresaron mediante la firma del consentimiento informado.

Criterios de exclusión

- Personas que no mostraron interés en participar en la investigación por lo que firmaron el consentimiento informado.

Se identificaron como variables de investigación las características generales de las personas, la presencia de afectación psicológica y el tipo de afectación psicológica. Dentro de las características generales de las personas se identificaron la edad, el sexo, la presencia de comorbilidades y el tipo de comorbilidades presentes. Dentro de los tipos de afectación psicológica se investigó en torno a la presencia de depresión, ansiedad, trastornos del sueño, cefalea, dolores musculares y la presencia de irritabilidad y cambios conductuales.

Se utilizaron como técnicas de investigación 
la revisión documental y la entrevista; la revisión documental permitió estudiar los referentes relacionados con las medidas restrictivas implementadas durante el periodo de confinamiento por la COVID-19. La entrevista posibilitó obtener información relacionada con las características generales de las personas investigadas y en relación con la presencia y tipo de afectación psicológica.

El cuestionario de investigación que se utilizó fue creado específicamente para el estudio, estuvo compuesto por un total de 19 preguntas en dos sesiones. La primera sesión incluyó 5 preguntas de respuestas múltiples orientadas a la identificación de las características generales de las personas y la segunda sesión, con 14 preguntas, se orientó hacia la identificación de la presencia y tipo de afectación psicológica. Al ser un cuestionario diseñado específicamente para la investigación se validó teniendo en cuenta la consistencia interna y la validez del mismo. Para evaluar la validez el instrumento fue sometido, previo a su aplicación, al juicio de un comité de expertos conformado por 7 especialistas en metodología de la investigación, psicología y medicina general. Se analizaron elementos relacionados con la actualización, coherencia, intencionalidad, consistencia, pertinencia, metodología, claridad, organización y objetividad entre otros. Después de una segunda ronda de revisión se autorizó su aplicación definitiva. Previo a su aplicación se realizó una prueba piloto en 15 personas para identificar $\mathrm{y}$ solucionar posibles errores semánticos que pudieran estar incluidos. Para el análisis de la consistencia interna se procedió a utilizar el coeficiente alfa de Cronbach. El resultado obtenido de 0,81 mostró una adecuada consistencia interna.

La información recopilada fue procesada automáticamente con la ayuda de programa estadístico SPSS en su versión 26 para Windows. Se determinaron medidas de tendencia central y de dispersión durante el procesamiento de las variables cuantitativas $\mathrm{y}$ frecuencias absolutas y por cientos en el caso de las variables cualitativas. Se definió el nivel de confianza en el $95 \%$ y el margen de error en el $5 \%$. Todos los resultados fueron expresados en forma de tablas estadísticas lo que facilitó la interpretación y comprensión de los mismos.

Durante el desarrollo del estudio se cumplieron los principios éticos estipulados en la Declaración de Helsinki 2 para realizar investigaciones en seres humanos. $\mathrm{La}$ participación en el estudio fue voluntaria, todos los participantes fueron informados de los métodos y objetivos del estudio. Los datos fueron utilizados únicamente con fines investigativos y se tomaron medidas de confiabilidad de la información que incluyeron la no utilización de datos de identidad personal, la utilización de códigos alfanuméricos y la eliminación de la base de datos con toda la información después de realizado el informe final de la investigación.

\section{RESULTADOS Y DISCUSIÓN}

La tabla I muestra la distribución de las características generales de las 247 personas investigadas. Destaca un promedio de edad de 51,07 años con una DE de 31,93 años. 
$(59,92 \%)$ en relación al sexo masculino $(40,08 \%)$. En relación con las comorbilidades se observa que el $38,87 \%$ de las personas refirieron al menos una comorbilidad asociada. La hipertensión arterial $(58,33 \%)$ fue la comorbilidad que con mayor frecuencia refirieron las personas investigadas; otras comorbilidades presentes fueron el hipotiroidismo $(28,12 \%)$, la diabetes mellitus $(16,67 \%)$ y la artritis reumatoide $(11,46 \%)$. También se identificaron 2 pacientes $(2,08 \%)$ con lupus eritematoso sistémico y con asma bronquial respectivamente.

TABLA I. Distribución de personas según características generales.

\begin{tabular}{|c|c|}
\hline $\begin{array}{c}\text { Características } \\
\text { generales }\end{array}$ & $\begin{array}{l}\text { Muestra total de } 247 \\
\text { personas } \\
\text { Frecuencia (por ciento) }\end{array}$ \\
\hline Promedio de edad & 51,07 años $*$ DE 31,93 \\
\hline \multicolumn{2}{|c|}{ Grupo de edades } \\
\hline Entre 20 y 39 años & $81(32,79)$ \\
\hline Entre 40 y 69 años & $109(44,13)$ \\
\hline De 60 años y más & $57(23,08)$ \\
\hline \multicolumn{2}{|c|}{ Sexo } \\
\hline Masculino & $99(40,08)$ \\
\hline Femenino & $148(59,92)$ \\
\hline \multicolumn{2}{|c|}{ Procedencia de comorbilidades } \\
\hline $\mathrm{Si}$ & $96(38,87)$ \\
\hline No & $151(61,13)$ \\
\hline \multicolumn{2}{|c|}{ Tipo de comorbilidades $n=96$ personas } \\
\hline Hipertensión arterial & $58(58,33)$ \\
\hline Diabetes mellitus & $16(16,67)$ \\
\hline Hipotiroidismo & $27(28,12)$ \\
\hline Artritis reumatoide & $11(11,46)$ \\
\hline $\begin{array}{l}\text { Lupus eritematoso } \\
\text { sistémico }\end{array}$ & $2(2,08)$ \\
\hline Asma bronquial & $2(2,08)$ \\
\hline
\end{tabular}

Se observa en la tabla II que el $72,47 \%$ de las personas encuestadas refirió haber presentado algún tipo de afectación psicológica durante el periodo que duró el confinamiento por la presencia de la COVID-19. El 27,53\% de los encuestados no refirió afectación psicológica. Dentro de las manifestaciones que con mayor frecuencia fueron reportadas se encuentran la ansiedad $(37,43 \%)$, los trastornos del sueño $(29,05 \%)$ y los dolores musculares $(27,37 \%)$. Otras manifestaciones de afectación psicológica referidas fueron la depresión (22,90\%), la cefalea $(17,88 \%)$ y la presencia de cambios conductuales (14,52\%). La irritabilidad fue la expresión de afectación psicológica que con menor frecuencia fue referida por las personas, aunque se presentó en el $10,06 \%$ de las personas.

TABLA II. Distribución de personas según presencia y tipo de afectación psicológica.

\begin{tabular}{|c|c|c|}
\hline \multirow{2}{*}{$\begin{array}{l}\text { Presencia y tipo de } \\
\text { afectación psicoló- } \\
\text { gica }\end{array}$} & \multicolumn{2}{|c|}{$\begin{array}{l}\text { Muestra total de } 247 \\
\text { personas }\end{array}$} \\
\hline & Frecuencia & Por ciento \\
\hline \multicolumn{3}{|c|}{ Presencia de afectación psicológica } \\
\hline $\mathrm{Si}$ & 179 & 72,47 \\
\hline No & 68 & 27,53 \\
\hline \multicolumn{3}{|c|}{ Tipo de afectación psicológica $n=179$} \\
\hline Depresión & 41 & 22,90 \\
\hline Ansiedad & 67 & 37,43 \\
\hline Trastornos del sueño & 52 & 29,05 \\
\hline Cefalea & 32 & 17,88 \\
\hline Dolores musculares & 49 & 27,37 \\
\hline Irritabilidad & 18 & 10,06 \\
\hline Cambios conductuales & 26 & 14,52 \\
\hline
\end{tabular}

Fuente: cuestionario de investigación

Varias fueron las causas fundamentales que identificaron las personas con alteraciones psicológicas como condicionantes de la presencia de estos trastornos (tabla III). La no existencia de esquemas terapéuticos y preventivos de la enfermedad fue referida por el $48,60 \%$ de las personas; el $26,82 \%$ refirió el desconocimiento de la enfermedad, el $26,26 \%$ refirió como causa la pérdida del 
puesto de trabajo y el $25,70 \%$ la presencia de problemas económicos. Otras causas identificadas fueron la muerte de algún familiar o amigo (21,79\%), la convivencia forzada $(17,88 \%)$ y la descompensación de enfermedad de base $(15,08 \%)$.

TABLA III. Distribución de personas según opinión relacionada con la causa que generó la afectación psicológica.

\begin{tabular}{lcc}
\hline \multicolumn{1}{c}{ Causas que generaron la presencia de afectación psicológica } & \multicolumn{2}{c}{ Muestra total 179 personas } \\
& Frecuencia & Por ciento \\
\hline $\begin{array}{l}\text { Desconocimiento de la enfermedad } \\
\text { La no existencia de esquemas terapéuticos y preventivos para la }\end{array}$ & 87 & 26,82 \\
enfermedad & & 48,60 \\
Muerte de familiar o amigo & 39 & 21,79 \\
Convivencia forzada & 32 & 17,88 \\
Pérdida de puesto de trabajo & 47 & 26,26 \\
Problemas económicos & 46 & 25,70 \\
Descompensación de enfermedad de base & 27 & 15,08 \\
\hline
\end{tabular}

Fuente: cuestionario de investigación

\section{Discusión}

La pandemia de COVID-19 fue el acontecimiento médico y social que condicionó el panorama mundial durante el año 2020; quizás su mayor expresión ocurrió durante los meses de marzo a junio en los cuales transcurrió un periodo de confinamiento obligatorio con medidas restrictivas que condicionaron las esferas sociales, económica, educativa y de salud entre otras (Rivero y Marrero, 2020). A partir de la nueva condición existente y teniendo en cuenta el panorama de salud existente las personas tuvieron que adaptarse forzosamente a una nueva manera de vivir; no todas las personas reaccionan de igual manera y los cambios adaptativos en algunas personas conlleva más tiempo que en otras (Ramos et al., 2021).

Durante el periodo de confinamiento se presentaron situaciones muy difíciles para muchas personas. De forma general la presencia de una nueva enfermedad, con elevados índices de contagio y muerte $y$ con el agravante del desconocimiento de la enfermedad y la no existencia de esquemas terapéuticos seguros para tratar a los enfermos y de medidas preventivas eficaces que garantizaran frenar el contagio son condiciones que generan ansiedad, irritabilidad y otros trastornos psicológicos (Carmenate y Rodríguez, 2020; OzamizEtxebarria, 2020).

En este sentido existen autores como Hernández Rodríguez (2020), que describen quelaCOVID-19haimpactadonegativamente en la salud mental no solo de los enfermos y familiares de las personas fallecidas; sino, en el resto de la población, que ha estado sometida a niveles altos de estrés durante un periodo prolongado de tiempo, sin contar con ayuda especializada. Rodríguez Cahill (2020) describe que existen retos, desde el punto de vista del abordaje psicológico, que deben de ser identificado y abordados para 'poder paliar la afectación psicológica secundaria a la COVID-19. 
Existen reportes que evidencia como la pérdida de la continuidad laboral y la aparición de problemas económicos como consecuencia del cierre de puestos de trabajo y de distintas fuentes de trabajo condicionaron la aparición de distintos tipos y grados de afectación psicológica (Chacón, Fernández y García, 2020; Peraza, 2020). En este sentido también es importante señalar que la pérdida de un familiar o amigo puede ser un suceso que necesita tiempo para ser asimilado. Durante el periodo de confinamiento hubo familias que perdieron varios miembros lo que trae consigo una carga emocional negativa mayor y por ende la reacción de las personas es diferente.

Era de esperar que manifestaciones clínicas de ansiedad, trastornos del sueño y depresión se contabilizaran dentro de las que con mayor frecuencia afectaron a las personas (Arias et al., 2020). Estas manifestaciones han sido sugeridas por otros autores como las de mayor afectación en frecuencia e intensidad como expresión clínica de los trastornos psicológicos (Martínez et al., 2020). Depende entonces de la integridad de los mecanismos de adaptación de cada persona para que estos trastornos psicológicos no prosperan a afectaciones más serias y consolidadas en las que pudiera entonces diagnósticas una enfermedad psiquiátrica (Pérez et al., 2020).

Si bien es cierto que aún continúa la pandemia de COVID-19, la situación epidemiológica ha cambiado sustancialmente en los últimos meses; se cuanta con varias vacunas que se están utilizando en muchos países a nivel mundial; ha disminuido el número de casos fatales aunque se mantiene el elevado índice de contagio y existe un mejor conocimiento de las formas clínicas, manifestaciones clínicas y complicaciones de la enfermedad. Sin embargo, la afectación psicológica, sobre toda en las personas que perdieron algún familiar o amigo, persisten a pesar del tiempo transcurrido.

Según la opinión del equipo de investigación es necesario que los gobiernos incentiven, activen y consoliden servicios de salud mental a nivel nacional que permitan afrontar con calidad la afectación psicológica sufrida durante el periodo de confinamiento por la COVID-19; este planteamiento coincide con los expresados en otras investigaciones como las realizadas por Rodríguez y colaboradores (2020) y Pérez y colaboradores (2020); resaltando el impacto negativo que ha generado la COVID-19 en la salud mental de la población y la necesidad de revertir esta situación a la mayor brevedad posible.

\section{CONCLUSIONES}

Se concluye que el periodo de confinamiento por la COVID-19 generó afectación psicológica en las personas pertenecientes a la parroquia Lizarzaburu de la ciudad de Riobamba. La ansiedad, los trastornos del sueño y los dolores musculares fueron los trastornos psicológicos que con mayor frecuencia fueron reportados. La no existencia de esquemas terapéuticos y preventivos de la enfermedad fue señalada como la principal causa para la aparición de trastornos psicológicos. Es necesario consolidar servicios de salud mental para hacer frente a las manifestaciones de afectación psicológicas generadas durante la fase de confinamiento 
obligatorio por la COVID-19.

\section{REFERENCIAS BIBLIOGRÁFICAS}

Arias Molina, Y., Herrero Solano, Y., Cabrera Hernández, Y., Guyat, D., \& Mederos, Y. (2020). Manifestaciones psicológicas frente a la situación epidemiológica causada por la COVID-19. Revista Habanera de Ciencias Médicas, 19(Supl. 1), e3350. Epub 10 de junio de 2020. Recuperado en 27 de marzo de 2021, de http:// scielo.sld.cu/scielo.php?script $=$ sci arttext\&pid $=$ S1729-519X202000040 $0012 \& \operatorname{lng}=\mathrm{es} \& \operatorname{lng}=\mathrm{es}$

Bermejo-Sánchez, F.R., Peña-Ayudante, W.R., \& Espinoza-Portilla, E. (2020). Depresión perinatal en tiempos del COVID-19: rol de las redes sociales en Internet. Acta Médica Peruana, 37(1), 88-93. https://dx.doi.org/10.35663/ amp.2020.371.913

Carmenate Rodríguez, I., \& Rodríguez Cordero, A. (2020). Repercusión psicológica en niños con Trastorno del espectro autista durante el confinamiento por COVID-19. MULTIMED, 24(3). Recuperado de http://www.revmultimed.sld.cu/ index.php/mtm/article/view/1978

Chacón-Fuertes, F., Fernández-Hermida, J.R., \& García-Vera, M.P. (2020). La Psicología ante la Pandemia de la COVID-19 en España. La Respuesta de la Organización Colegial. Clínica y Salud, 31(2), 119-123. Epub 27 de julio de 2020.https://dx.doi. org/10.5093/clysa2020a18

Hernández Rodríguez, J. (2020). Impacto de la COVID-19 sobre la salud mental de las personas. Medicentro ElectróNica, 24(3), 578-594. Recuperado de http://medicentro.sld.cu/index.php/ medicentro/article/view/3203

Leon Reyna, P., Lora Loza, M., \& Rodriguez Vega, J. (2021). Relación entre estilo de vida y estrés laboral en el personal de enfermería en tiempos de COVID-19. Revista Cubana de Enfermería, 37(1). Recuperado de http://revenfermeria.sld.cu/index. php/enf/article/view/4043

Martínez Pérez, A., Fernández-Fernández, V., Alcántara-López, M., LópezSoler, C., \& Castro Sáez, M. (2020). Preliminary results of the impact of COVID-19 on children and adolescents exposed to intrafamily abuse. Terapia psicológica, 38(3), 427-445. https://dx.doi.org/10.4067/ S0718-48082020000300427

Martínez-Pérez, J., Rivas-Laguna, Y., Bermudez-Cordoví, L., GutiérrezFavier, E., \& Rivero-Rodríguez, I. (2020). Efectos de la COVID-19 sobre estados afectivos emocionales de la población adulta de Puerto Padre. Revista Electrónica Dr. Zoilo E. Marinello Vidaurreta, 45(6). Recuperado de http:// revzoilomarinello.sld.cu/index.php/ zmv/article/view/2388 
Ozamiz-Etxebarria, N. (2020). Niveles de estrés, ansiedad y depresión en la primera fase del brote del COVID-19 en una muestra recogida en el norte de España. Cadernos de Saúde Pública,36,(4),e00054020. Recuperado de: https://doi. org/10.1590/0102-311X00054020

Peraza de Aparicio, X. (2020). Salud laboral frente a la pandemia del COVID-19 en Ecuador. MediSur, 18(3), 507-511. Epub 02 de junio de 2020. Recuperado en 27 de marzo de 2021, de http:// scielo.sld.cu/scielo.php? script $=$ sci_ arttext\&pid $=$ S1727-897X202000030 $0507 \& \operatorname{lng}=\mathrm{es} \& \mathrm{t} \operatorname{lng}=\mathrm{pt}$

Pérez Abreu, M.R., Gómez Tejeda, J.J., Tamayo Velázquez, O., Iparraguirre Tamayo, A.E., \& Besteiro Arjona, E.D. (2020). Alteraciones psicológicas en estudiantes de medicina durante la pesquisa activa de la COVID-19. MEDISAN, 24(4), 537-548. Epub 08 de julio de 2020. Recuperado en 27 de marzo de 2021, de http://scielo.sld.cu/scielo. php?script $=$ sci_arttext\&pid=S102930192020000400537\&lng=es\&tlng= es

Ramos Remedios, T., Mendivia Ramos, T., Mendivia Ramos, T., Hernández Pérez, A., \& Pérez Salgado, I. (2021). Alteraciones psicológicas en estudiantes de Medicina durante la pesquisa activa de la COVID-19. Revista de Ciencias Médicas de Pinar del Río, 25(2), e4606. Recuperado de http://revcmpinar.sld.cu/index.php/ publicaciones/article/view/4606

Rivero Arias, E., \& Marrero Santos, M. (2020). Alteraciones psicológicas en profesionales de la salud durante la pandemia de la COVID-19. Prevención desde una Misión Médica. Revista Cubana de Salud y Trabajo, 21(3), 53-58. Recuperado de http:// www.revsaludtrabajo.sld.cu/index. php/revsyt/article/view/175

Rodríguez-Morales, A.J., Sánchez-Duque, J.A., Hernández Botero, S., PérezDíaz, C.E., Villamil-Gómez, W.E., Méndez, C.A., et al. (2020). Preparación y control de la enfermedad por coronavirus 2019 (COVID-19) en América Latina. Acta Médica Peruana, 37(1), 3-7. https://dx.doi. org/10.35663/amp.2020.371.909

Rodríguez Cahill, C. (2020). Cinco retos psicológicos de la crisis del COVID-19. Journal of Negative and No Positive Results, 5(6), 583588. Recuperado de https://dx.doi. org/10.19230/jonnpr.3662

Rodríguez Rodríguez, T., Fonseca Fernández, M., Valladares González, A.M., \& López Angulo, L.M. (2020). Protocolo de actuación psicológica ante la COVID-19 en centros asistenciales. Cienfuegos. Cuba. MediSur, 18(3), 368-380. Epub 02 de junio de 2020. Recuperado en 27 de marzo de 2021, de http://scielo.sld.cu/scielo. php? script $=$ sci_arttext\&pid $=$ S 1727 897X2020000300368\&lng=es\&tlng 
$=\mathrm{pt}$

Solis Cartas, U., \& Martinez Larrarte, J.P. (2020). Therapeutic options to cytokine release syndrome in patients with COVID-19. Revista Cubana de Medicina Militar, 49(3), e783. Epub 25 de noviembre de 2020. Recuperado en 09 de marzo de 2021, de http://scielo.sld.cu/scielo. php?script $=$ sci_arttext\&pid=S0138$65572020000300022 \& \operatorname{lng}=\mathrm{es} \& \operatorname{tlng}=$ en

Solis Cartas, U. (2020). Coronaviruses and rheumatic diseases, assumptions, myths and realities. Revista Cubana de Reumatología, 22(2), e791. Epub 01 de agosto de 2020. Recuperado en 09 de marzo de 2021, de http://scielo.sld.cu/scielo. php?script=sci_arttext\&pid=S181759962020000200001\&lng=es\&tlng= en 